\title{
Muertes por desnutrición en América del Sur en los últimos veinte años
}

\author{
Deaths due to Malnutrition in South America in the last Twenty Years
}

Gloria Deossa Restrepo ${ }^{1}$, Luis Fernando Restrepo Betancur ${ }^{2}$, John Edison Velásquez Vargas ${ }^{3}$

\section{Resumen}

Introducción. El hambre es uno de los principales referentes en la política mundial de seguridad alimentaria. Sin embargo, cada año mueren miles de personas a causa de la falta de ingesta de alimentos. Objetivo. Evaluar el número de fallecimientos por desnutrición en América del Sur, en un periodo correspondiente a los últimos veinte ańos de información. Materiales y métodos. Se empleó el modelo lineal general, incorporando la prueba de Tukey con base en un nivel de significancia estadístico del 5\%. Adicionalmente, se llevó a cabo la técnica multivariada clúster. Resultados. El mayor número de muertes por desnutrición ocurrió en los niños menores de cuatro años y en adultos mayores. El mayor porcentaje de muertes por desnutrición en niños menores de un año se presentó en Venezuela (23,9\% mujeres; $25,4 \%$ hombres). Chile tiene el menor porcentaje de muertes por desnutrición en menores de un año ( $0,4 \%$ mujeres; $0,5 \%$ hombres). Conclusión. A pesar de que los países de América del Sur presentan gran disponibilidad de alimentos, las muertes por hambre siguen siendo prevalentes en parte de la región.

Palabras claves: Alimentación, desnutrición, disponibilidad, estadísticas, fallecimientos, hambre.

\footnotetext{
1. Profesora Asociada, Universidad de Antioquia, Medellín, Colombia. Magíster en Ciencias de la Alimentación y Nutrición Humana ORCID: https://orcid.org/0000-0002-1635-1601

2. Docente Titular Universidad de Antioquia, Medellín, Colombia. Estadístico. Especialista en Estadística. Grupo STATISTICAL. ORCID: http://orcid.org/0000-0002-8583-5028

3. Nutricionista Dietista. Magíster en Salud Colectiva. Universidad Federal de Mato Grosso, Brasil.

ORCID: https://orcid.org/0000-0001-6379-5400

Correspondencia: gloria.deossa@udea.edu.co
} 


\section{Abstract}

Introduction. Hunger is one of the main issues in the world food security policy, however, thousands of people die every year due to lack of food intake. Objective. To evaluate the number of deaths due to malnutrition in South America, in a period corresponding to the last twenty years of information. Materials and methods. The general linear model was used, incorporating the Tukey test based on a level of statistical significance of 5\%. Additionally, the multivariate cluster technique was carried out. Results. The highest number of deaths due to malnutrition occurred in children under four years of age and in older adults. The highest percentage of deaths due to malnutrition in children under one year old occurred in Venezuela (23.9\% women; $25.4 \%$ men). Chile has the lowest percentage of deaths due to malnutrition in children under one year of age ( $0.4 \%$ women; $0.5 \%$ men). Conclusion. Although the countries of South America have high food availability, starvation deaths remain prevalent in part of the region.

Keywords: Food, malnutrition, availability, statistics, deaths, hunger.

\section{Introducción}

Todos los seres humanos, sin distinción de nacionalidad, raza, sexo, origen nacional o étnico, color, religión, lengua, entre otras condiciones, tenemos los mismos derechos, sin diferencia alguna. Los derechos humanos están contemplados en todas las leyes de cada Estado y deben ser garantizados por éste (1). Con la aprobación de la Declaración Universal de los Derechos Humanos el 16 de noviembre de 1974, se comienza a hablar sobre la erradicación del hambre y la malnutrición. En el artículo 25 se establece "Toda persona tiene derecho a un nivel de vida adecuado que le asegure, así como a su familia, la salud y el bienestar y en especial la alimentación" (2).

En la Cumbre Mundial de Alimentación de 1996, en Roma, los países representan- tes por invitación de la Organización de Naciones Unidas (ONU), se estableció el acceso a los alimentos sanos y nutritivos como derecho a una alimentación adecuada y derecho fundamental de toda persona a no padecer hambre. Es así, entonces, que la alimentación es un derecho fundamental, y como tal, los Estados tienen la obligación de asegurar que ninguno de sus habitantes muera de hambre. Es por esto que el Estado no puede ser pasivo en el reconocimiento de los derechos y debe ser activo en la protección de éstos (3).

La Organización Mundial de la Salud (OMS) define la desnutrición como el desequilibrio celular entre el suministro de nutrientes, la energía y la demanda requerida por el cuerpo, con el fin de garantizar el crecimiento de la persona, el mantenimiento y el desarrollo cabal de sus funciones (4). 
De acuerdo con el informe de la seguridad alimentaria y nutrición en el mundo, presentado por la UNICEF en el año 2017, se encuentra que a partir del año 2000 se ha reducido la prevalencia de desnutrición en todas las regiones del mundo, siendo más evidente en África Meridional y Occidental, Asia Central, Sureste de Asia y Suramérica. Sin embargo, el norte de África ha presentado un pequeño incremento, pasando del 6,8\% en el año 2000 al 8,3\% en el 2016 y Oceanía del 5,3\% en el 2000 a 6,8\% en el 2016. A pesar de la disminución en las cifras de desnutrición, uno de cada cuatro nińos en el mundo presenta retraso en el crecimiento, lo cual incrementa el riesgo de alteraciones en la capacidad cognitiva, lo que repercute en el aumento de enfermedades infecciosas. En 2016 se estimó que el número de habitantes en el mundo con subnutrición estaba en 815 millones (5).

La desnutrición materna e infantil aporta más de un tercio de defunciones infantiles, lo que representa más del $10 \%$ del total de morbilidad en el mundo, siendo el mayor contribuyente de la mortalidad infantil, de acuerdo a un estudio de 2008 de la Organización Panamericana de la Salud (6). En el mismo informe, se destaca el retraso en el crecimiento en Perú, el cual se estimó en $29,8 \%$, presentando alta variabilidad en diferentes departamentos (oscilando entre $6,7 \%$ en Tacna y $60,1 \%$ en Huancavelica). La Encuesta de demografía y de salud de Nigeria, efectuada en 2003 detectó que el $38 \%$ de niños menores de cinco ańos en ese país presentaban retraso en el crecimiento debido a factores asociados a la desnutrición (7).

En Colombia, 42,7\% de la población vive en condiciones de inseguridad alimentaria, lo cual indica que casi la mitad de los habitantes carecen de las condiciones para tener acceso a una alimentación saludable. Los departamentos de Colombia que presentan mayores índices de inseguridad alimentaria son Nariño $(67,7 \%)$, Chocó $(64,2 \%)$, Sucre $(62,9 \%)$, Bolívar $(61,7 \%)$ y Magdalena $(61,4 \%)$. Lo anterior evidencia que la región atlántica y la zona pacífica son las más afectadas por falta de condiciones que permitan suplir las necesidades mínimas de alimentación. (ENSIN 2010) (8). En el año 2016, el Instituto Nacional de Salud (INS) (9) reportó la muerte por desnutrición de nińos menores de cinco ańos pertenecientes a la comunidad "Wayuu" (Colombia); lo anterior asociado a la corrupción política que desvía los recursos económicos destinados a las comunidades. En Antioquia, para el año 2000, la tasa de muertes debidas a desnutrición fue de 22,7 por cada 100.000 menores de edad (10).

La situación de desnutrición también se evidencia en personas mayores. En un estudio llevado a cabo en Dinamarca, se reportó que una tercera parte de adultos mayores ingresados a salas geriátricas presentaban condiciones de desnutrición, debido a depresión, tratamientos médicos inadecuados y baja ingesta calórica (11). La desnutrición principalmente en personas mayores y en adolescentes, incrementa la morbimortali- 
dad, los días de estancia hospitalaria y los costos asociados (12). Un estudio llevado a cabo en Holanda encontró que la desnutrición tenía un impacto en factores relacionados a los ataques cardiacos, resfriados frecuentes y esquizofrenia (13).

Diversas investigaciones han mostrado una correlación entre la pobreza y la morbilidad. En Argentina se detectó una relación directamente proporcional entre el número de menores de edad fallecidos con el analfabetismo, la falta de un trabajo fijo estable y la capacidad económica reducida de las familias y en forma inversa con la calidad del agua (14).

El objetivo de este estudio fue evaluar el porcentaje de fallecimientos por desnutrición en los diferentes países de América del Sur, en los últimos veinte años.

\section{Materiales y métodos}

Se utilizó la base de datos relacionada con las muertes por desnutrición, reportada por la Organización Panamericana (PAHO), complementándose con las bases de datos de disponibilidad alimentaria y PIB provenientes de la plataforma FAOSTAT. Se empleó el modelo lineal general con el fin de efectuar los contrastes entre países de América del Sur, incorporando la prueba de Tukey con base en un nivel de significancia estadístico del 5\%. Adicionalmente, se llevó a cabo la técnica de clúster, la cual tiene como fin clasificar a los países en temas relacionados con el PIB asignado a salud, disponibilidad de los principales alimentos y muertes por desnutrición. Se complementó con análisis descriptivo comparativo para las variables de índole cuantitativa. Se utilizó el paquete estadístico SAS UNIVERSITY y R versión 3.0.0.

Las variables evaluadas fueron: número de muertes por desnutrición en los países de América del Sur, disponibilidad de frutas, hortalizas, legumbres, carne y leche expresadas en g/persona/día. Adicionalmente se evaluó el porcentaje del PIB en salud. Como variables control, se tuvieron en cuenta el país y los rangos de edad.

\section{Resultados}

Al efectuar el análisis estadístico para cada país de América del Sur, el mayor porcentaje de muertes por desnutrición en niños menores de un año se presentó en Venezuela (23,9\% mujeres; $25,4 \%$ hombres), seguido de Paraguay (17\% mujeres; $22,5 \%$ hombres) y Colombia (15,9\% mujeres; $22,5 \%$ hombres). Chile tiene el menor porcentaje de muertes por desnutrición en menores de un año $(0,4 \%$ mujeres; $0,5 \%$ hombres $)$. Venezuela, Paraguay y Colombia comandan la estadística de muertes por desnutrición en niños menores de cinco años. Uruguay, Argentina y Chile, son las naciones que presentan las más altas estadísticas de muertes por desnutrición en adultos mayores de 70 años, caso contrario de Colombia que posee los porcentajes menores. Entre los cinco y 
cincuenta años de edad, en general, se pre- dad por desnutrición en los países de Amésentan las estadísticas más bajas de mortali- rica del Sur (ver Cuadro 1).

Cuadro 1. Porcentaje de muertes por desnutrición por país, rango de edad y sexo.

\begin{tabular}{|c|c|c|c|c|c|c|c|c|c|c|c|c|c|c|}
\hline \multirow[b]{3}{*}{ País } & \multicolumn{14}{|c|}{ Rango de edad } \\
\hline & \multicolumn{2}{|c|}{$<1$} & \multicolumn{2}{|c|}{$1-4$} & \multicolumn{2}{|c|}{$5-9$} & \multicolumn{2}{|c|}{$10-14$} & \multicolumn{2}{|c|}{$15-19$} & \multicolumn{2}{|c|}{$20-24$} & \multicolumn{2}{|c|}{$25-29$} \\
\hline & $\mathbf{M}$ & $\mathbf{H}$ & $\mathbf{M}$ & $\mathbf{H}$ & $\mathbf{M}$ & $\mathbf{H}$ & $\mathbf{M}$ & $\mathbf{H}$ & $\mathbf{M}$ & $\mathbf{H}$ & $\mathbf{M}$ & $\mathbf{H}$ & $\mathbf{M}$ & $\mathbf{H}$ \\
\hline Argentina & 4,0 & 5,8 & 4 & 4,2 & 1 & 0,8 & 1 & 0,6 & 1 & 0,8 & 1 & 1 & 1 & 1 \\
\hline Bolivia & 6,3 & 6,9 & 8 & 9,8 & 1 & 0,8 & 2 & 3,0 & 2 & 1,7 & 2 & 4 & 3 & 4 \\
\hline Brasil & 10 & 11 & 4 & 4,2 & 1 & 1,1 & 1 & 0,9 & 1 & 1,5 & 2 & 2 & 2 & 2 \\
\hline Chile & 0,4 & 0,5 & 1 & 0,5 & 1 & 0,3 & 1 & 0,4 & 1 & 0,5 & 1 & 1 & 1 & 1 \\
\hline Colombia & 16 & 19 & 9 & 9,2 & 2 & 1,9 & 1 & 1,4 & 1 & 1,5 & 2 & 2 & 1 & 2 \\
\hline Ecuador & 10 & 13 & 9 & 9,6 & 2 & 1,7 & 1 & 1,8 & 2 & 2,0 & 2 & 2 & 2 & 2 \\
\hline Paraguay & 17 & 23 & 11 & 12 & 2 & 2 & 2 & 1,8 & 1 & 1,5 & 1 & 1 & 1 & 1 \\
\hline Perú & 5,7 & 7,4 & 7 & 8,3 & 2 & 2,1 & 1 & 1,8 & 1 & 1,8 & 2 & 2 & 2 & 2 \\
\hline Uruguay & 3,3 & 4,8 & 1 & 1,4 & 0 & 0,1 & 0 & 0,4 & 1 & 0,8 & 1 & 1 & 1 & 1 \\
\hline \multirow[t]{2}{*}{ Venezuela } & 24 & 25 & 20 & 17 & 3 & 2,5 & 2 & 1,7 & 2 & 1,9 & 2 & 2 & 2 & 2 \\
\hline & \multicolumn{2}{|c|}{$30-34$} & \multicolumn{2}{|c|}{$35-39$} & \multicolumn{2}{|c|}{$40-44$} & \multicolumn{2}{|c|}{$45-49$} & \multicolumn{2}{|c|}{$50-54$} & \multicolumn{2}{|c|}{ 55-59 } & \multicolumn{2}{|c|}{$60-64$} \\
\hline País & $\mathbf{M}$ & $\mathbf{H}$ & $\mathbf{M}$ & $\mathbf{H}$ & $\mathbf{M}$ & $\mathbf{H}$ & $\mathbf{M}$ & $\mathbf{H}$ & $\mathbf{M}$ & $\mathbf{H}$ & $\mathbf{M}$ & $\mathbf{H}$ & $\mathbf{M}$ & $\mathbf{H}$ \\
\hline Argentina & 0,7 & 0,7 & 1 & 0,8 & 1 & 0,9 & 1 & 1,6 & 2 & 2,5 & 2 & 4 & 3 & 6 \\
\hline Bolivia & 2,7 & 2,3 & 3 & 3,4 & 5 & 3,9 & 4 & 3,4 & 5 & 3,9 & 5 & 5 & 5 & 5 \\
\hline Brasil & 1,6 & 2,2 & 2 & 2,5 & 2 & 3,2 & 3 & 3,6 & 3 & 4,0 & 3 & 5 & 4 & 5 \\
\hline Chile & 0,3 & 0,9 & 1 & 1,0 & 1 & 1,7 & 1 & 2,4 & 2 & 2,7 & 2 & 4 & 2 & 5 \\
\hline Colombia & 1,3 & 1,5 & 2 & 1,7 & 2 & 2,0 & 2 & 2,5 & 2 & 2,6 & 2 & 3 & 3 & 4 \\
\hline Ecuador & 1,8 & 1,9 & 2 & 1,9 & 2 & 2,1 & 2 & 2,0 & 2 & 2,4 & 2 & 3 & 3 & 3 \\
\hline Paraguay & 0,6 & 0,7 & 2 & 1,4 & 2 & 2,0 & 1 & 1,6 & 2 & 2,6 & 2 & 3 & 2 & 4 \\
\hline Perú & 1,8 & 1,9 & 2 & 1,9 & 2 & 2,2 & 2 & 2,5 & 3 & 2,8 & 3 & 3 & 4 & 4 \\
\hline Uruguay & 0,7 & 0,5 & 1 & 1,3 & 1 & 0,7 & 1 & 2,0 & 1 & 3,3 & 3 & 3 & 3 & 5 \\
\hline \multirow[t]{2}{*}{ Venezuela } & 1,6 & 1,6 & 2 & 1,7 & 2 & 2,3 & 2 & 2,3 & 1 & 2,5 & 2 & 2 & 2 & 3 \\
\hline & \multicolumn{2}{|c|}{$65-69$} & \multicolumn{2}{|c|}{$70-74$} & \multicolumn{2}{|c|}{$75-79$} & \multicolumn{2}{|c|}{$80-84$} & \multicolumn{2}{|c|}{$85-89$} & & & & \\
\hline País & $\mathbf{M}$ & $\mathbf{H}$ & $\mathbf{M}$ & $\mathbf{H}$ & $\mathbf{M}$ & $\mathbf{H}$ & $\mathbf{M}$ & $\mathbf{H}$ & $\mathbf{M}$ & $\mathbf{H}$ & $\mathbf{M}$ & $\mathbf{H}$ & $\mathbf{M}$ & $\mathbf{H}$ \\
\hline Argentina & 4,4 & 7,8 & 7 & 11 & 12 & 15 & 17 & 16 & 20 & 13 & 13 & 7 & 5 & 2 \\
\hline Bolivia & 6,9 & 6,8 & 11 & 9,8 & 12 & 11 & 9 & 7,8 & 7 & 5,6 & 3 & 2 & 1 & 1 \\
\hline Brasil & 4,7 & 6,6 & 7,0 & 8,7 & 9,6 & 9,9 & 13 & 10 & 13 & 8,8 & 9,5 & 5,2 & 6 & 3 \\
\hline Chile & 4,4 & 7,5 & 6,4 & 11,1 & 11 & 15 & 18 & 18 & 22 & 15,6 & 18 & 8,9 & 9 & 3 \\
\hline Colombia & 4,0 & 5,2 & 6,1 & 7,5 & 8,7 & 8,9 & 11 & 9,7 & 11 & 8,3 & 8,3 & 5,0 & 5 & 2 \\
\hline Ecuador & 3,2 & 4,7 & 5 & 5,9 & 7,0 & 8,2 & 10 & 11 & 14 & 9,8 & 12 & 7,8 & 8 & 5 \\
\hline Paraguay & 2,3 & 4,0 & 4,1 & 5,4 & 7,0 & 6,5 & 12 & 8,4 & 13 & 9,3 & 11 & 5,4 & 5 & 3 \\
\hline Perú & 4,9 & 5,8 & 7,4 & 8,3 & 10 & 11 & 12 & 12 & 13 & 10,3 & 8,8 & 6,1 & 7 & 3 \\
\hline Uruguay & 3,2 & 7,6 & 7,5 & 11,0 & 12 & 14 & 14 & 17 & 22 & 14 & 17 & 8,0 & 6 & 3 \\
\hline Venezuela & 2,7 & 4,2 & 3,6 & 5,1 & 5,1 & 6,2 & 7,3 & 6,8 & 7,7 & 5,3 & 5 & 2,8 & 3 & 2 \\
\hline
\end{tabular}

Fuente: Elaboración propia con base en la información reportada por la FAO. M= mujeres; H=hombres. 
Venezuela es el país donde se presentan los mayores reportes de muertes por desnutrición en niños menores de cinco años, con diferencia estadísticamente significativa, con respecto a los demás países del sur del continente $(\mathrm{p}<0,05)$. En el rango de edad entre los cinco y cincuenta ańos de edad no se presentaron diferencias estadísticas significativas $(p>0,05)$. Argentina, Chile y Uruguay se diferencian respecto a las demás naciones en relación a muertes por desnutrición en personas mayores de 64 años, como se puede apreciar en el Cuadro 2.

Cuadro 2. Análisis comparativo entre países por rango de edad, relacionado con el porcentaje de muertes por desnutrición.

\begin{tabular}{|c|c|c|c|c|c|c|c|}
\hline \multirow[b]{2}{*}{ País } & \multicolumn{7}{|c|}{ Rango de edad } \\
\hline & $<1$ & $1-4$ & $5-9$ & $10-14$ & $15-19$ & $20-24$ & $25-29$ \\
\hline Argentina & $\mathrm{d}$ & c & $\mathrm{a}$ & $\mathrm{a}$ & $\mathrm{a}$ & $\mathrm{a}$ & $\mathrm{a}$ \\
\hline Bolivia & d & $\mathrm{b}$ & $\mathrm{a}$ & $\mathrm{a}$ & $\mathrm{a}$ & $\mathrm{a}$ & $\mathrm{a}$ \\
\hline Brasil & c & c & $\mathrm{a}$ & $\mathrm{a}$ & $\mathrm{a}$ & $\mathrm{a}$ & $\mathrm{a}$ \\
\hline Chile & $\mathrm{e}$ & $\mathrm{d}$ & $\mathrm{a}$ & $\mathrm{a}$ & $\mathrm{a}$ & $\mathrm{a}$ & $\mathrm{a}$ \\
\hline Colombia & $\mathrm{b}$ & $\mathrm{b}$ & $\mathrm{a}$ & $\mathrm{a}$ & $\mathrm{a}$ & $\mathrm{a}$ & $\mathrm{a}$ \\
\hline Ecuador & c & $\mathrm{b}$ & $\mathrm{a}$ & $\mathrm{a}$ & $\mathrm{a}$ & $\mathrm{a}$ & $\mathrm{a}$ \\
\hline Paraguay & $\mathrm{b}$ & $\mathrm{b}$ & $\mathrm{a}$ & $\mathrm{a}$ & $\mathrm{a}$ & $\mathrm{a}$ & $\mathrm{a}$ \\
\hline Perú & $\mathrm{d}$ & $\mathrm{c}$ & a & $\mathrm{a}$ & $\mathrm{a}$ & $\mathrm{a}$ & $\mathrm{a}$ \\
\hline Uruguay & $\mathrm{d}$ & $\mathrm{d}$ & $\mathrm{a}$ & $\mathrm{a}$ & $\mathrm{a}$ & $\mathrm{a}$ & $\mathrm{a}$ \\
\hline Venezuela & $\mathrm{a}$ & $\mathrm{a}$ & $\mathrm{a}$ & $\mathrm{a}$ & a & $\mathrm{a}$ & $\mathrm{a}$ \\
\hline País & $30-34$ & 35-39 & $40-44$ & $45-49$ & $50-54$ & 55-59 & $60-64$ \\
\hline Argentina & $\mathrm{a}$ & $\mathrm{a}$ & $\mathrm{a}$ & $\mathrm{a}$ & $\mathrm{b}$ & $\mathrm{b}$ & $\mathrm{a}$ \\
\hline Bolivia & $\mathrm{a}$ & $\mathrm{a}$ & a & $\mathrm{a}$ & $\mathrm{a}$ & $\mathrm{a}$ & $\mathrm{a}$ \\
\hline Brasil & $\mathrm{a}$ & $\mathrm{a}$ & $\mathrm{a}$ & $\mathrm{a}$ & $\mathrm{b}$ & $\mathrm{b}$ & $\mathrm{a}$ \\
\hline Chile & $\mathrm{a}$ & $\mathrm{a}$ & a & $\mathrm{a}$ & $\mathrm{b}$ & $\mathrm{b}$ & $\mathrm{a}$ \\
\hline Colombia & $\mathrm{a}$ & $\mathrm{a}$ & $\mathrm{a}$ & $\mathrm{a}$ & $\mathrm{b}$ & $\mathrm{b}$ & $\mathrm{a}$ \\
\hline Ecuador & $\mathrm{a}$ & $\mathrm{a}$ & $\mathrm{a}$ & $\mathrm{a}$ & $\mathrm{b}$ & $\mathrm{b}$ & $\mathrm{a}$ \\
\hline Paraguay & $\mathrm{a}$ & $\mathrm{a}$ & $\mathrm{a}$ & $\mathrm{a}$ & $\mathrm{b}$ & $\mathrm{b}$ & $\mathrm{a}$ \\
\hline Perú & $\mathrm{a}$ & $\mathrm{a}$ & $\mathrm{a}$ & $\mathrm{a}$ & $\mathrm{b}$ & $\mathrm{b}$ & $\mathrm{a}$ \\
\hline Uruguay & $\mathrm{a}$ & $\mathrm{a}$ & $\mathrm{a}$ & $\mathrm{a}$ & $\mathrm{b}$ & $\mathrm{a}$ & $\mathrm{a}$ \\
\hline Venezuela & $\mathrm{a}$ & $\mathrm{a}$ & a & $\mathrm{a}$ & $\mathrm{b}$ & $\mathrm{b}$ & $\mathrm{a}$ \\
\hline País & 65-69 & $70-74$ & $75-79$ & $80-84$ & $85-89$ & $90-94$ & $>94$ \\
\hline Argentina & $\mathrm{a}$ & a & $\mathrm{a}$ & $\mathrm{a}$ & $\mathrm{a}$ & $\mathrm{a}$ & a \\
\hline Bolivia & $\mathrm{b}$ & $\mathrm{b}$ & $\mathrm{b}$ & $\mathrm{b}$ & $\mathrm{d}$ & d & d \\
\hline Brasil & $\mathrm{a}$ & $\mathrm{b}$ & $\mathrm{c}$ & $\mathrm{a}$ & c & c & c \\
\hline Chile & $\mathrm{a}$ & $\mathrm{a}$ & $\mathrm{a}$ & $\mathrm{a}$ & $\mathrm{a}$ & $\mathrm{a}$ & $\mathrm{a}$ \\
\hline Colombia & $\mathrm{a}$ & $\mathrm{b}$ & $\mathrm{c}$ & $\mathrm{b}$ & $\mathrm{c}$ & c & $\mathrm{c}$ \\
\hline Ecuador & $\mathrm{b}$ & b & c & $\mathrm{b}$ & $\mathrm{b}$ & $\mathrm{b}$ & $\mathrm{b}$ \\
\hline Paraguay & $\mathrm{b}$ & c & $\mathrm{c}$ & $\mathrm{b}$ & $\mathrm{c}$ & $\mathrm{c}$ & c \\
\hline Perú & $\mathrm{a}$ & $\mathrm{b}$ & $\mathrm{b}$ & $\mathrm{b}$ & $\mathrm{b}$ & $\mathrm{b}$ & $\mathrm{b}$ \\
\hline Uruguay & $\mathrm{a}$ & $\mathrm{a}$ & $\mathrm{a}$ & $\mathrm{a}$ & $\mathrm{a}$ & $\mathrm{a}$ & $\mathrm{a}$ \\
\hline Venezuela & $\mathrm{b}$ & c & $\mathrm{d}$ & c & $\mathrm{d}$ & $\mathrm{d}$ & $\mathrm{d}$ \\
\hline
\end{tabular}

Fuente: Elaboración propia. Letras distintas indican diferencia estadística significativa $(\mathrm{p}<0,05)$. 
El análisis clúster relacionado con el número de defunciones por malnutrición permitió definir cuatro grupos, así: uno conformado por Brasil y Venezuela, el siguiente por: Bolivia, Paraguay y Uruguay, el tercer grupo está definido por Argentina, Colombia y Perú, mientras que el cuarto clúster se asoció con Chile y Ecuador. En lo relacionado con la disponibilidad de alimentos se definieron cuatro grupos de países, así: grupo 1 conformado por Bolivia y Venezuela; grupo 2 conformado por Perú, Chile, Paraguay; grupo 3 conformado por Argentina; grupo 4 conformado por Colombia, Uruguay, Ecuador y Brasil. Al evaluar el gasto en salud expresado en porcentaje del PIB, Ecuador, Bolivia, Perú, Venezuela forman un clúster, otro lo conforma Uruguay, otro grupo lo establecen Paraguay, Brasil y Chile, mientras Argentina y Colombia, forman otro grupo (ver Cuadro 3).

Cuadro 3. Análisis clúster, relacionado con: muertes por desnutrición, disponibilidad de alimentos y PIB para salud.

\begin{tabular}{|c|c|c|c|c|}
\hline \multicolumn{5}{|c|}{ Muertes por desnutrición } \\
\hline País & Clúster 1 & Clúster 2 & Clúster 3 & Clúster 4 \\
\hline \multicolumn{5}{|l|}{ Argentina } \\
\hline \multicolumn{5}{|l|}{ Bolivia } \\
\hline \multicolumn{5}{|l|}{ Brasil } \\
\hline \multicolumn{5}{|l|}{ Chile } \\
\hline \multicolumn{5}{|l|}{ Colombia } \\
\hline \multicolumn{5}{|l|}{ Ecuador } \\
\hline \multicolumn{5}{|l|}{ Paraguay } \\
\hline \multicolumn{5}{|l|}{ Perú } \\
\hline \multicolumn{5}{|l|}{ Uruguay } \\
\hline \multicolumn{5}{|l|}{ Venezuela } \\
\hline \multicolumn{5}{|c|}{ Disponibilidad de alimentos } \\
\hline País & Clúster 1 & Clúster 2 & Clúster 3 & Clúster 4 \\
\hline \multicolumn{5}{|l|}{ Argentina } \\
\hline \multicolumn{5}{|l|}{ Bolivia } \\
\hline \multicolumn{5}{|l|}{ Brasil } \\
\hline \multicolumn{5}{|l|}{ Chile } \\
\hline \multicolumn{5}{|l|}{ Colombia } \\
\hline \multicolumn{5}{|l|}{ Ecuador } \\
\hline \multicolumn{5}{|l|}{ Paraguay } \\
\hline \multicolumn{5}{|l|}{ Perú } \\
\hline \multicolumn{5}{|l|}{ Uruguay } \\
\hline Venezuela & & & & \\
\hline
\end{tabular}




\begin{tabular}{|c|c|c|c|c|}
\hline \multicolumn{5}{|c|}{ PIB Salud } \\
\hline País & Clúster 1 & Clúster 2 & Clúster 3 & Clúster 4 \\
\hline Argentina & & & & \\
\hline Bolivia & & & & \\
\hline Brasil & & & & \\
\hline Chile & & & & \\
\hline Colombia & & & & \\
\hline Ecuador & & & & \\
\hline Paraguay & & & & \\
\hline Perú & & & & \\
\hline Uruguay & & & & \\
\hline Venezuela & & & & \\
\hline
\end{tabular}

Fuente: Elaboración propia.

En lo relacionado con la disponibilidad de frutas (g/persona/día), Colombia ocupa el primer puesto, seguido de Ecuador, Brasil y Chile, mientras que Bolivia es el de menor participación. En hortalizas (g/persona/ día), Chile, Argentina y Paraguay son los países que poseen los más altos valores de esta disponibilidad, mientras que Brasil, Colombia y Ecuador presentan las estadísticas más bajas. En cuanto a la disponibilidad de carne (g/persona/día), es Argentina el país con mayor disponibilidad de este producto, mientras que en el otro extremo se encuentra Perú, país con las menores cifras de disponibilidad per cápita. Con respecto a la leche, son Brasil, Argentina, Colombia y Uruguay los que encabezan la mayor disponibilidad y Bolivia ocupa el último lugar (ver Cuadro 4).

Cuadro 4. Análisis descriptivo y comparativo, relacionado con la disponibilidad de alimentos (g/persona/día).

\begin{tabular}{|c|c|c|c|c|c|}
\hline Pais & Frutas & Hortalizas & Legumbres & Carne & Leche \\
\hline \multicolumn{5}{|c|}{ Media \pm STD } \\
\hline Argentina & $34,5 \pm 4,7 \mathrm{~d}$ & $107,1 \pm 2,6 \mathrm{~b}$ & $3,0 \pm 1,5 \mathrm{~g}$ & $152 \pm 4,4 \mathrm{a}$ & $244,5 \pm 51 \mathrm{~b}$ \\
\hline Bolivia & $20,5 \pm 7,2 \mathrm{e}$ & $80,4 \pm 22,1 \mathrm{~d}$ & $6,0 \pm 1,2 \mathrm{f}$ & $55,7 \pm 6 \mathrm{~b}$ & $78,8 \pm 11,4 \mathrm{~g}$ \\
\hline Brasil & $61,9 \pm 5,1 \mathrm{a}$ & $59,7 \pm 7,9 \mathrm{e}$ & $45,2 \pm 1,7 \mathrm{a}$ & $99 \pm 5,9 \mathrm{c}$ & $311 \pm 22,4 \mathrm{a}$ \\
\hline Chile & $54,3 \pm 7,6 \mathrm{~b}$ & $150,4 \pm 9,6 \mathrm{a}$ & $11,2 \pm 1,5 \mathrm{e}$ & $58 \pm 2,2 \mathrm{c}$ & $160,3 \pm 16 \mathrm{e}$ \\
\hline Colombia & $68,9 \pm 5,9 \mathrm{a}$ & $46,3 \pm 3,8 \mathrm{e}$ & $19,3 \pm 1,2 \mathrm{c}$ & $45,5 \pm 3 \mathrm{c}$ & $326 \pm 11,3 \mathrm{a}$ \\
\hline Ecuador & $65,3 \pm 9,4 \mathrm{a}$ & $47,5 \pm 6,2 \mathrm{e}$ & $7,2 \pm 1,4 \mathrm{f}$ & $43 \pm 3,6 \mathrm{c}$ & $237,0 \pm 4,4 \mathrm{~d}$ \\
\hline Paraguay & $40,2 \pm 1,4 \mathrm{c}$ & $103,6 \pm 9,9 \mathrm{bc}$ & $29,5 \pm 5,7 \mathrm{~b}$ & $56,2 \pm 27 \mathrm{c}$ & $177,4 \pm 9,1 \mathrm{~d}$ \\
\hline Perú & $53,9 \pm 3,6 \mathrm{~b}$ & $91,2 \pm 15,6 \mathrm{~cd}$ & $19,6 \pm 1,7 \mathrm{c}$ & $10 \pm 0,6 \mathrm{~d}$ & $117,2 \pm 5,5 \mathrm{f}$ \\
\hline Uruguay & $43,1 \pm 6,9 \mathrm{c}$ & $93,5 \pm 5,6 \mathrm{c}$ & $8,3 \pm 1,0 \mathrm{f}$ & $85,6 \pm 27 \mathrm{~b}$ & $298 \pm 101 \mathrm{a}$ \\
\hline Venezuela & $44,6 \pm 4,6 \mathrm{c}$ & $87,7 \pm 8,9 \mathrm{~d}$ & $15,9 \pm 3,2 \mathrm{~d}$ & $52,5 \pm 11 \mathrm{c}$ & $142,5 \pm 54 \mathrm{e}$ \\
\hline
\end{tabular}

Fuente: Elaboración propia. 


\section{Discusión}

Junto con la obesidad y el sobrepeso, considerados la pandemia del siglo XXI, coexiste aún la desnutrición en muchos países (15). En nuestro estudio, se aprecia que son las mujeres las más afectadas por la desnutrición y son los grupos de edad de menos de un año y los de más de 70 años quienes presentan las mayores frecuencias de muerte atribuibles a esta causa, cuyos principales desencadenantes son la pobreza y el subdesarrollo, tal como lo han demostrado varios investigadores en diferentes países; y cuya consecuencia se evidencia en la inseguridad alimentaria severa, que para el caso de las mujeres en América Latina se encuentra en $8,4 \%$ y en $6,9 \%$ para los hombres (16).

Existe gran heterogeneidad en las situaciones socioeconómicas en los países que conforman la parte del continente americano estudiado y son diversas las situaciones de salud, de producción, de vulnerabilidad alimentaria, de etapas de transición demográfica y epidemiológica que se presentan, entre los países y al interior de ellos. De igual forma, en los países de esta región se aprecian alteraciones en la ingesta de alimentos que se asocian a carencias de zinc, hierro, yodo y vitamina $\mathrm{A}$, entre otros, que coexisten con exceso en el consumo de azúcares simples y grasas saturadas, responsables, entre otros factores, del incremento de las enfermedades crónicas no trasmisibles (17).

El grado de desarrollo de una nación se mide por la tasa de mortalidad infantil, además de otros factores, la cual refleja directa o indirectamente las condiciones de vida y el estado sanitario de una población y debe ser analizada con prudencia (18). A lo largo de la historia se han presentado cambios en los comportamientos en cuanto a mortalidad infantil entre los países de Latinoamérica. Por ejemplo, en el siglo XX la mortalidad infantil era 2,4 veces mayor en Chile que en Argentina, para el siglo XXI la mortalidad en menores de un año en Argentina es casi el doble que la reportada en Chile. El factor ambiental es uno de los determinantes más influyentes en las condiciones nutricionales y de mortalidad infantil (17). Las cifras más altas de desnutrición y mortalidad infantil ocurren en países donde la agricultura se ve afectada constantemente por desastres naturales. De igual forma, las condiciones higiénico-sanitarias de la vivienda influyen directamente en el estado nutricional. Según Rosaneli et al, los más vulnerables a padecer hambre y desnutrición son las personas pobres, de zonas rurales, zonas urbanas y víctimas de desastres naturales, con bajo nivel educativo y con acceso limitado a acueducto y alcantarillado (19). Dado lo anterior, en las políticas públicas deberían priorizarse a las personas quienes viven bajo estas condiciones, con el fin de brindar apoyo o buscar mejorar las condiciones susceptibles de cambio. Otros factores biológicos para mejorar la condición de desnutrición en los países tienen que ver con el estado nutricional de las gestantes, las condiciones de la lactancia y de alimentación complementaria, aspectos importantes a tener en 
cuenta para mejorar la situación de desnutrición infantil y evitar las muertes por esta causa (20-22).

Entre las consecuencias que se derivan de la desnutrición infantil, además del alto riesgo de mortalidad, con cifras hasta del $60 \%$, se destaca la posibilidad de contraer enfermedades infecciosas como la diarrea, la malaria, la neumonía y el sarampión (61\%, 57\%, 53\% y $45 \%$ respectivamente) y en los sobrevivientes de la desnutrición, se incrementa en la edad adulta, de manera significativa, el riesgo de presentar enfermedades crónicas no trasmisibles (23). Por lo anterior, se deben hacer esfuerzos de inversión de capital económico por parte de los países para prevenirla, pues el impacto de esta condición en la morbimortalidad genera una pérdida importante de capital humano, con efectos sociales y económicos contundentes a corto y largo plazo, tanto en la educación como en la productividad. Todos los seres humanos deben tener derecho a la alimentación, deber que los gobiernos deben cumplir. Pese a esto, en Colombia $54,2 \%$ de la población vive en condiciones de inseguridad alimentaria, limitando el acceso a una alimentación saludable, con regiones más afectadas como la Atlántica y la Pacífica, donde es necesario focalizar políticas públicas, para mejorar las condiciones de estos habitantes (8).

Es preocupante que se sigan presentando muertes por desnutrición en menores de 5 años, ya que estas se encuentran asociadas a los determinantes sociales, económicos y políticos de salud, pese a los avances tecnológicos, a la gran disponibilidad de alimentos en la región y al incremento en los desperdicios de alimentos que se presentan (24).

La gran disponibilidad de alimentos que se encuentran en América del Sur y la falta de políticas acordes a la planeación y distribución de estos a los más necesitados, hace que se vivan fenómenos como los que se presentan hoy en día, donde las carencias de nutrientes son comunes en las personas más desfavorecidas haciendo más prevalentes las muertes por desnutrición en los grupos poblacionales extremos, es decir los menores de 5 años y los mayores de 70 ańos (25).

Las muertes por desnutrición en adultos plantean desafíos para los profesionales de la salud por lo que es importante visibilizar esta población tan vulnerable, atendiendo a retos para la construcción de políticas sociales y de salud que cubran las necesidades y mejoren la calidad de vida. En un estudio realizado en Colombia que tenía como objetivo comparar la mortalidad de personas adultas mayores por deficiencias nutricionales, se encontró que las muertes fueron más prevalentes en las mujeres $35,4 \%$ frente a los hombres 33,4\% (26). Resultados muy similares a los nuestros, pues al comparar la muerte en adultos mayores en los diferentes países, se encontró mayor prevalencia de muertes en mujeres en todos los países, incluyendo a Colombia. 
El hambre, una de las principales amenazas para la vida, se debe erradicar, de acuerdo con la política mundial de seguridad alimentaria; sin embargo, cada año mueren miles de personas por esta causa. América del Sur es un referente de gran disponibilidad y variedad de alimentos y no es ajena a las muertes por desnutrición debidas a inequidades en salud, determinantes sociales y económicos y cambio climático; factores que ameritan análisis por dirigentes gubernamentales, quienes deben reflexionar al momento de elaborar sus planes de gobierno, políticas y acuerdos, pues estos influyen en la salud y en la vida de los seres humanos (27-28).

\section{Conclusión}

Pese a que los países de América del Sur presentan gran disponibilidad de alimentos, las muertes por hambre siguen siendo prevalentes en la mayor parte del continente como se evidenció en el estudio. Estas se deben en parte a la inseguridad alimentaria severa que se presenta en América Latina y que es más prevalente en las mujeres, al igual que a la pobreza y al subdesarrollo.

Las políticas públicas deben buscar el mejoramiento de la seguridad alimentaria en la población menos favorecida, con bajo nivel educativo y con acceso limitado a acueducto y alcantarillado, con una consideración especial hacia el sexo femenino.

Se deben aunar esfuerzos por mejorar el estado nutricional del binomio madre/hijo, hacer campańas enfocadas a promocionar la lactancia exclusiva hasta los seis meses, fomentar la introducción de alimentación complementaria de manera adecuada y velar por el derecho a una alimentación digna y adaptada a las condiciones de salud de los adultos mayores. De este modo se contribuirá al logro de uno de los objetivos de desarrollo sostenible, que dice "hambre cero"; y así, evitar muertes por desnutrición en los seres humanos.

Declaración de conflicto de intereses: los autores declararon no tener conflicto de intereses.

\section{Referencias}

1. Naciones Unidas, ¿Qué son los Derechos Humanos? 2018 [Sitio en internet], Disponible en línea en: https://www,ohchr,org/SP/Issues/Pages/WhatareHumanRights,aspx Consulta: 27 de abril de 2019

2. 2. Organización de las Naciones Unidas (ONU), Declaración universal de derechos Humanos, En: Asamblea General en su resolución 217 A (III); 1948,1-9.

3. Absjorn E, El derecho humano a una alimentación adecuada y a no padecer hambre, Doctrina, 2003; 1(1): 43-46.

4. World Health Organization, WHO child growth standards and the identification of severe acute malnutrition in infants and children 2009, World Health Organization; 2009.

5. FAO, IFAD, UNICEF, WFP and WHO, The State of Food Security and Nutrition in the World 2017, Building resilience for peace and food security, Rome, FAO,2017. 
6. Pan American Health Organization ( PAHO)"Malnutrition in infants and young children in Latin America and the Caribbean: Achieving the Millennium Development Goals" Washington, D,C, PAHO,2008.

7. Ajieroh V, A Quantitative Analysis of Determinants of Child and Maternal Malnutrition in Nigeria, IFPRI Nigeria Strategy Support Program Brief No, $11,2010$.

8. Instituto Colombiano de Bienestar Familiar (ICBF), Encuesta Nacional de la Situación Nutricional en Colombia (ENSIN), 2010, Bogotá, Colombia, 2011.

9. Instituto Nacional de Salud (2016) [Internet], "Desnutrición infantil en Colombia: Marco de referencia”, [Consultado el 8 de Marzo de 2018], Disponible en: https://www,minsalud,gov,co/sites/rid/ Lists/BibliotecaDigital/RIDE/DE/AS/papeles-salud-n3,pdf

10. Toro J, Cardona D, Mortalidad por desnutrición en menores de cinco años: cinco años antes y después de la implementación del Programa mana, Antioquia, 1998-2007, Rev, Fac,Nac, Salud Pública 2013; 31(1): 93-101.

11. Bamia C, Halkjaer J, Lagiou P, Trichopoulos D, Tjønneland A, Berentzen TL, et al, Weight change in later life and risk of death amongst the elderly: the European Prospective Investigation into Cancer and Nutrition-Elderly Network on Ageing and Health study, J Intern Med 2010;268(2):133e44.

12. Correia MI, The impact of malnutrition on morbidity, mortality, length of hospital stay and costs evaluated through a multivariate model analysis, Clin Nutr 2003;22(3):235e9.

13. Rooij SR, Blunted cardiovascular and cortisol reactivity to acute psychological stress: a summary of results from the Dutch famine birth cohort study, Int J Psychophysiol 2013;90:21e7, 471-473.

14. Tafani R, Gaspio N, Pobreza, enfermedad y muerte en Argentina, Rev Salud Pública, 2009;13(1):18-32.
15. Córdova JA, La obesidad: la verdadera pandemia del siglo xxi Cirugía y Cirujanos, 2016;84(5):351-355.

16. FAO, OPS, WFP y UNICEF, 2018, Panorama de la seguridad alimentaria y nutricional en América Latina y el Caribe 2018, Santiago.

17. CEPAL, UNICEF: Desnutrición infantil en América Latina y el Caribe, Revista Desafíos,2006; 2(1).

18. Giachetto G. Mortalidad infantil en Uruguay: una mirada crítica. Arch. Pediatr. Urug. 2010; 81(3):139-140.

19. Rosaneli C, Ribeiro A, Assis L, Silva T, Siqueira J. La fragilidad humana frente a la pobreza y el hambre. Rev. Bioét. 2015;23( 1 ): 89-97.

20. Belfort Murillo D, García A, Ligner P, Factores de riesgo relacionados a la desnutrición en niños menores de 5 años, del centro de salud San Juan octubre 2018-abril 2019, BS thesis, BABAHOYO: UTB, 2019.

21. Calero Ruiz D, et al, Percepción ante situaciones de desastres y plan de respuesta familiar con enfoque en nutrición en las comunidades "Roberto Lara" y "Valle del Volcán", municipio de Nindirí, Masaya, Período Septiembre a diciembre 2017, Diss, Universidad Nacional Autónoma de Nicaragua, Managua, 2018.

22. Bernal, L., Consuelo Bernal, M., \& Gollop, T. (2018). Amniocentesis precoz y biopsia de vellosidad corial. Pérdidas fetales y anomalías congénitas en un grupo de gestantes brasileñas. NOVA, 16(29), 51-61. Disponible en: https://revistas.unicolmayor. edu.co/index.php/nova/article/view/841

23. Lafuente Y, Rodríguez S, Fontaine V, Yáñez R, Prevalencia de la desnutrición crónica en niños menores de 5 ańos atendidos en el Centro de Salud Tacopaya, primer semestre gestión 2014, Gac Med Bol, 2016;39 (1): 26-29.

24. Behm Rosas H, Determinantes económicos y sociales de la mortalidad en América Latina, Revista Cubana de Salud Pública, 2017; 43:287-312. 
25. Dommarco J, La doble carga de la mala nutrición en América Latina y las políticas para enfrentarla, Archivos Latinoamericanos de Nutrición, 2015; 65(Suplemento 1).

26. Cardona D, Segura A, Espinosa AM, Mortalidad de adultos mayores por deficiencias nutricionales en los departamentos de Colombia, Rev Salud Pública, 2012; 14:584-97.

27. FAO y OPS, Panorama de la Seguridad Alimentaria y Nutricional en América Latina y el Caribe, Santiago de Chile, 2017.

28. Parada-Rico DA, López-Guerrero N, Martínez-Laverde M. Bajo peso al nacer y su implicación en el desarrollo psicomotor. Rev. cienc. cuidad. 2015;12(2):87-99. https://doi. org/10.22463/17949831.511 